\title{
Design of CNC Pneumatic Measuring Instrument Monitoring System Based on Configuration Collaborative
}

\author{
YANG Zeqing ${ }^{1, a}$, FAN Min ${ }^{1, b}$,LIU Libing ${ }^{1, c}$ and JIN Wei ${ }^{2, d}$ \\ ${ }^{1}$ School of Mechanical Engineering, Hebei University of Technology, Tianjin \\ 2 JiangSuRuipu Machine Tool Co.,Ltd., Jiangsu \\ ayzq1982@126.com, bfm13820986967@126.com, ${ }^{\mathrm{c}}$ 13478334105@163.com, \\ d643647276@qq.com
}

\begin{abstract}
Keywords: pneumatic measuring instrument; $\mathrm{CNC}$; configuration collaborative; monitoring; KINGVIEW.

Abstract:In view of the problems that the off-line inspection of traditional measuring instrument was inconvenient to automatically correct the Computer Numerical Control (CNC) machining process parameters and feedback control the in-process machining quality, the configuration collaborative monitoring method was proposed based on the analysis of the working principle and technological process of the $\mathrm{CNC}$ pneumatic measuring instrument. The real-time monitoring of the measuring instrument's measuring process was realized by means of the interaction between the configuration unit and the intermodule information. And then the pneumatic measuring instrument monitoring system was designed based on KINGVIEW and PLC, which can realize the real-time monitoring and controlling of measuring probe in manual mode, semi-automatic mode and automatic mode, overtravel and collision warning, procedure allowance feedback controlling, workpiece quality statistical analysis and so on. The system provides the basis for the monitoring of processing quality of batch manufacturing, as well as health maintenance equipment timely finding hidden trouble.
\end{abstract}

\section{Introduction}

Traditionally, manual measurement, off-line measurement and on-line measurement have been used to measure the workpiece dimension of $\mathrm{CNC}$ batch manufacturing. Traditional manual measurement and off-line measurement are convenient, but the production efficiency is low. On-line measurement mode which integrates the measuring instrument into the $\mathrm{CNC}$ machine tool and the production line can realize the measurement of the critical dimensions of the workpiece without any downtime between the processes. On-line measurement mode can avoid the problem of measurement deviation caused by the secondary loading of workpiece and improve the production efficiency, and it is especially suitable for full automatic mass production line and intelligent workshop operation ${ }^{[1]}$. Therefore, it is important to complete the measuring task safely and efficiently. Configuration monitoring provides a function of real-time multi-task monitoring field operation and is widely used in automation process industry control ${ }^{[2-3]}$, Wang et al.[4] designed the transformer monitoring system based on the KINGVIEW, which can automatically obtain the running parameters and status, and send control commands; Gyan et al.[5] applied the configuration software to the dynamic process monitoring of the hydrogen cooling generator to monitor the parameters such as liquid level and pressure; Chen et al.[6] used the iFIX configuration software to remotely monitor the state of the hydraulic stents to realize automation and integration of industrial production. Morsi et al.[7]developed a monitoring system to realize real-time monitoring of oil refining process and provide guarantee for safe production by using configuration software.

Based on the thought of configuration modular design and the analysis of working principle and technological process of CNC pneumatic measurement instrument, KINGVIEW is used to design monitoring system and using the monitoring system is convenient to monitor the production process and detect the hidden trouble in time. 


\section{Working principle of $\mathrm{CNC}$ pneumatic measuring instrument}

$\mathrm{CNC}$ pneumatic measuring instrument in CNC milling machining center is composed of measuring device, driving device and controller. The measuring device which converts the variation of the measured parameters into electrical signals by differential transformer type displacement sensor consists of probe, measuring bar and measuring head ${ }^{[8]}$. According to the measurement device, the structure has the single arm Vnimar measuring device(hereinafter referred to as the $\mathrm{V}$ head) and the dual-arm Unimar measuring instrument(hereinafter referred to as the $U$ head), corresponding to the end surface measurement and the radial measurement respectively. The measuring device is drove by driving device to enter or exit the measuring station, and the probe is aimed at the center or suitable position by adjusting the micro-adjusting mechanism. The controller is composed of signal conditioning module, microprocessor module and display module, which is connected with the measuring device, driving device and the I/O interface of machine tool controller. The working principle of the pneumatic measuring instrument is shown in figure 1.First, the calibration of the measuring device was zeroed and the ratio of the measuring device was adjusted by measuring the standard parts in the manual mode. Then the controller sends instructions to realize manual, semi-automatic and automatic measurement of workpiece size. When measuring, the controller sends the control signal, so that the driving device drives the measuring device into the measuring station, the probe is in contact with the surface of the workpiece, resulting in relative displacement, and then the differential transformer displacement transducer converts the displacement to the output of the electrical signal. The signal conditioning module amplifies, filters and modulates the electrical signal and transmits to the microprocessor for data processing. In automatic mode, parameters are set through the display module, after data processing and parameter analysis and comparison, the signal is sent to the CNC milling machining center, and then automatic control milling feeding.

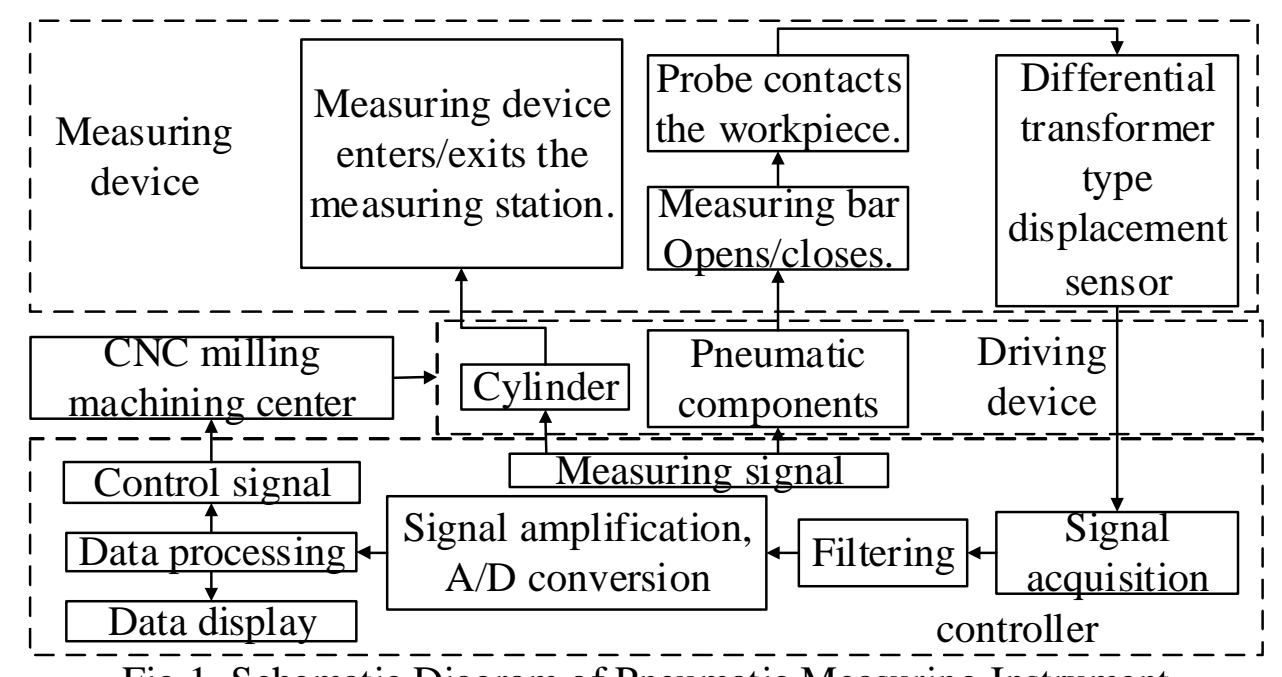

Fig. $\overline{1}$. Schematic Diagram of Pneumatic Measuring Instrument

\section{The method of measuring process monitoring based on configuration collaborative}

In view of the limitations of traditional configuration monitoring, for example, the monitoring parameters cannot be dynamically adjusted, and the flexibility and interactivity of the configuration elements are poor, the configuration collaborative monitoring method was proposed. Fig 2 shows the design idea of this method. The real-time monitoring of the measuring instrument's measuring process was realized by means of the interaction between the configuration unit and the intermodule information. The monitoring objects can be monitored in a collaborative manner according to different monitoring tasks by configurating data, interface and parameter configuration.

Configuration collaborative is the process of combining the configuration unit into the whole monitoring system. Synergy is mainly manifested as the goal synergy, that is, in order to achieve a 
monitoring task, each unit needs to cooperate with each other. The information interaction between modules mainly means that the monitoring data of the upper process will inevitably affect the processing of the next process; hence the monitoring results produced in this process is processed and compared to determine the processing requirements of the next process, and the processing parameters of machine tool are adjusted in a timely manner to monitor the processing process.

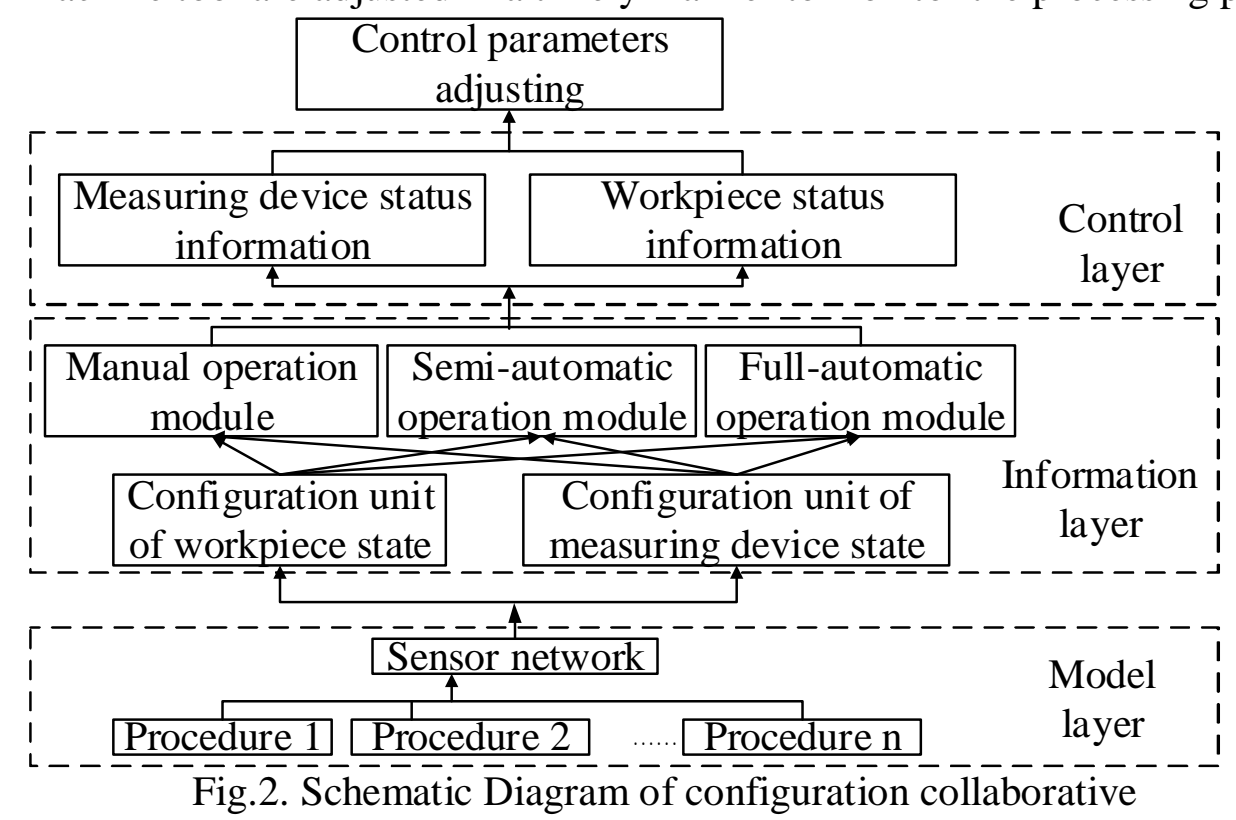

\section{Design of monitoring system for $\mathrm{CNC}$ pneumatic measuring instrument}

\section{Hierarchy of the monitoring system}

Real-time monitoring and controlling of measuring device, display of measurement results, overtravel and collision warning, alarm of overtravel and collision, procedure allowance feedback controlling, control mode automatically switching, and workpiece quality statistical analysis and so on should be realized by the monitoring system.

According to the design idea of configuration collaborative, the hierarchical chart of pneumatic measuring instrument monitoring system is shown in Fig.3, including the equipment layer, control layer and monitoring layer.According to the monitoring requirements of measuring device, Siemens S7-300 PLC was adopted to realize the automatic detection and control of the analog quantity and digital quantity, and the monitoring layer was composed of the upper computer monitor software developed by the KINGVIEW to realize the monitoring of the operation status of the measuring device and the display of the dimension parameters of the workpiece.

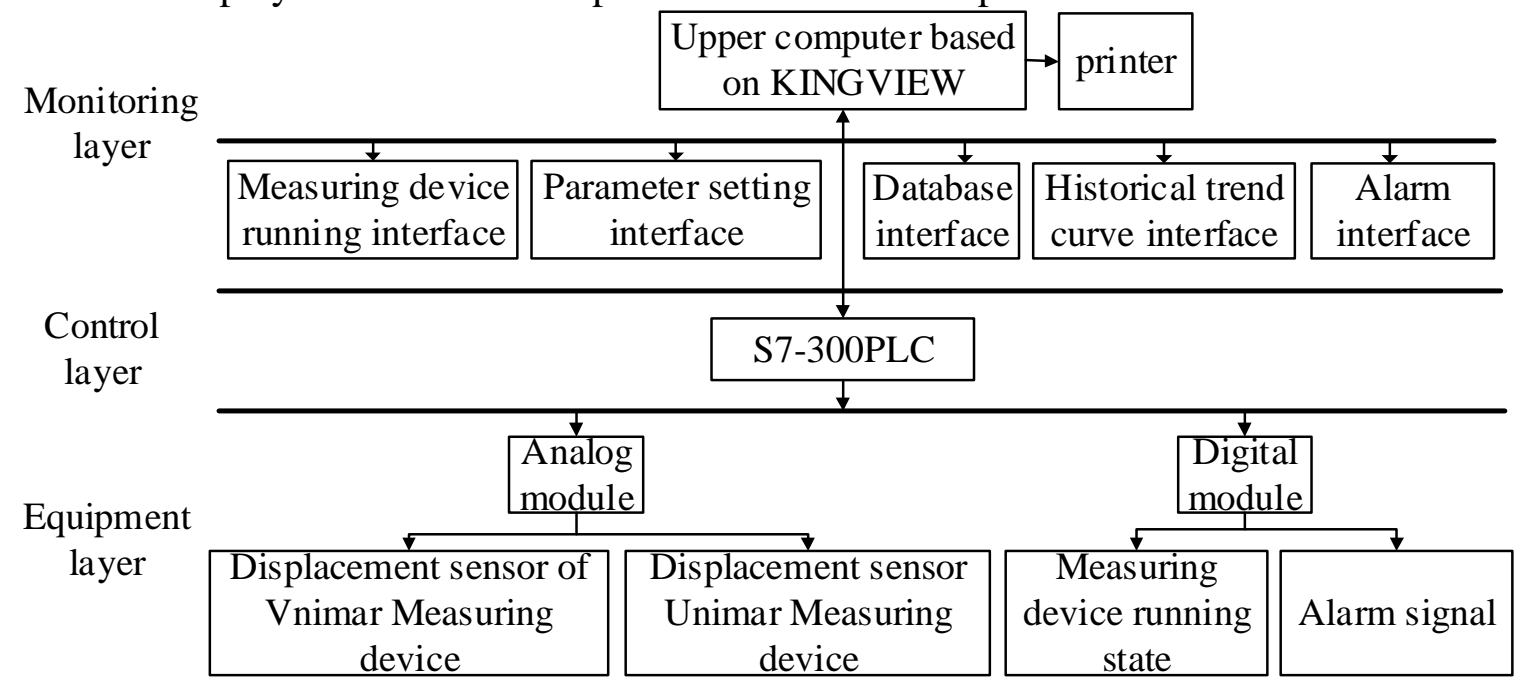

Fig.3. Hierarchical Chart of Monitoring System 


\section{Design of control layer}

According to the working principle and monitoring requirements of the CNC pneumatic measuring instrument, the control objects of the monitoring system were defined as the movement state of Vnimar measuring instrument and Unimar measuring instrument and process parameter and so on. This is the control flow chart shown in Fig.4. The initialization procedure was applied to initialize the parameters; the manual control subroutine was required to meet the motion control requirements of the measuring instrument, such as forward, backward, open and close; The automatic control subroutine was required to meet the continuous motion requirements of the measuring instrument, so the actual running distance of the measuring instrument was compared with limit value to ensure the continuity of the measurement. In addition, the sampled data was filtered in PLC, and the processing result and movement state were transmitted to the upper machine to display; PLC and upper computer transmit data through RS232.

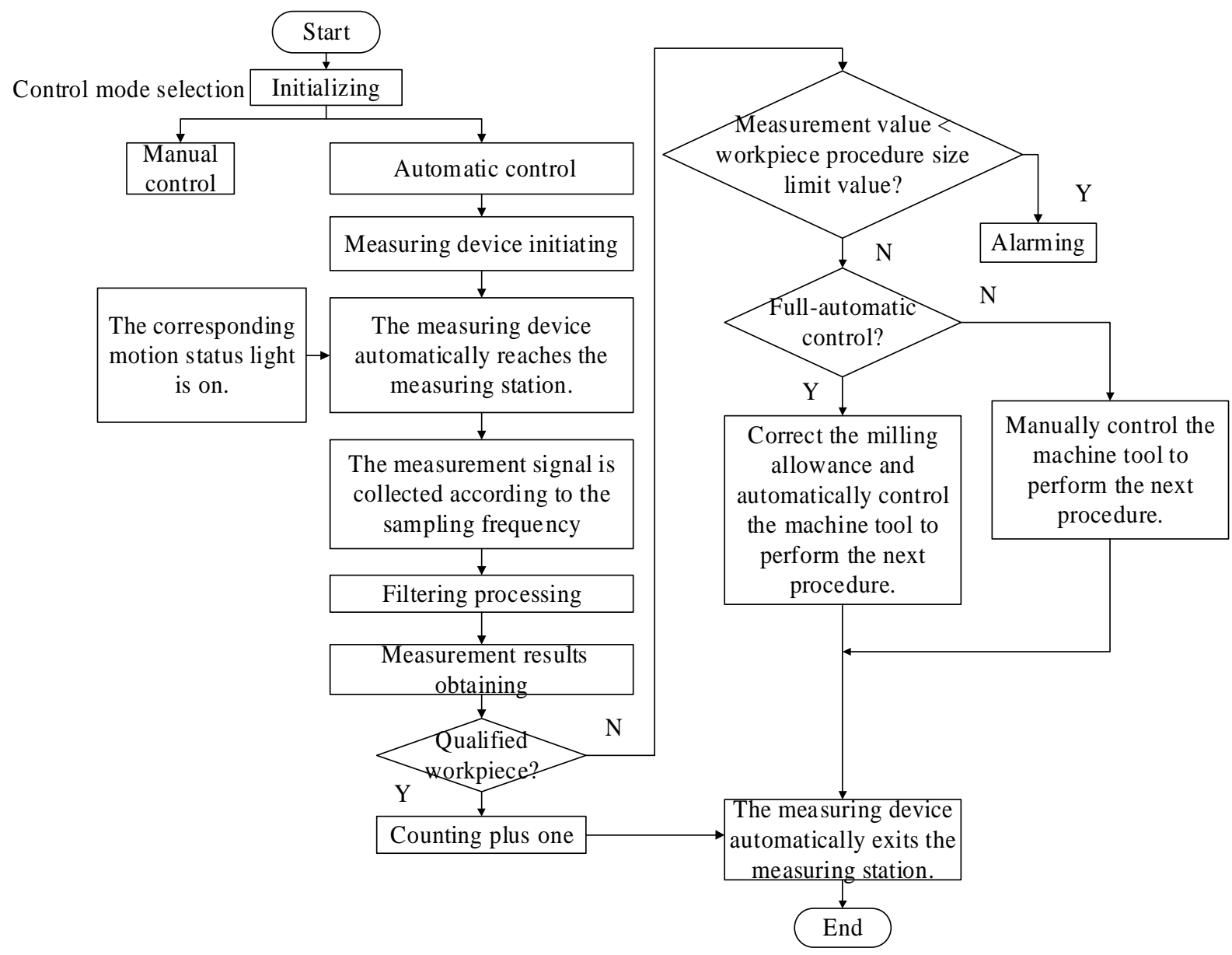

Fig.4.Control Flow Chart

\section{Design of monitoring layer}

The upper computer monitoring software mainly carried out real-time monitoring and display of measuring processes of Vnimar and Unimar measuring device and process parameters in the manual, semi-automatic and full-automatic modes to realize the visualization of production process.

\section{Establishment of a data dictionary}

Based on the analysis of the state of monitoring object and monitoring parameter, measuring processes of Unimar and Vnimar measuring device are abstracted into coordinate transformation, and the data dictionary was set up and mapped to the program. The output parameters of displacement sensor are collected and transmitted by PLC.I/O variables were defined to represent the movement coordinates of measuring device and the measured size of the workpiece and the standard workpiece. 
To facilitate the statistical analysis of the machining quality of the workpiece, designing a database to store the measuring results of workpiece, the measuring condition of measuring device and so on.

\section{Parameter setting module}

The parameter setting module is the premise and foundation for the operation of the monitoring system, mainly including the setting of error compensation value, signal point and overtravel limit value. The error compensation value was obtained by comparing the theoretical value of the standard workpiece which can be used to correct the subsequent measurement results. The signal point was set to meet the machining accuracy requirements of different processing stages. The corresponding signal light was on When the milling allowance of the workpiece conforms to a certain numerical interval, so as to prompt the operator to carry out subsequent operation tasks; To prevent the measuring device from running the overtravel, the overtravel limit was set.

\section{Signal processing technology}

Filtering technique was used to filter the sampled data to reduce the influence of the interference signal on the accuracy of the measurement results ${ }^{[9]}$. When measuring in the same position, the change in data was smaller, and interference was mostly pulse interference and periodic interference, so that the filtering method of the median value filtering, median value average filtering and the limit amplitude filtering combined with the slip average filtering were set. In addition, the sensor measurement results were processed through the software algorithm to solve the nonlinear problem between the input and output of the measurement system ${ }^{[10]}$; hence the intelligent correction of the nonlinear system was realized by using the curve fitting method.

\section{Monitoring module of measuring device}

The monitoring module of measuring device was divided into the monitoring module of Vnimar measuring device and Unimar measuring device, and the monitoring moudle of Unimar measuring device shown in fig. 5 is used as an example. The monitoring module was composed by operation mode switching, measuring movement monitoring and measuring results display. The undisturbed switching of manual mode, semi-automatic mode and full-automatic mode, as well as the selection of filtering mode was controlled by the module of operation mode switching; The dynamic real-time monitoring and display of the measuring process in three working modes were realized by the module of measuring movement monitoring; The position of the measuring instrument, the real-time voltage value, the geometry size of the workpiece, the process status, the number of qualified workpieces, and the measurement action indicator are shown in the measuring results display.

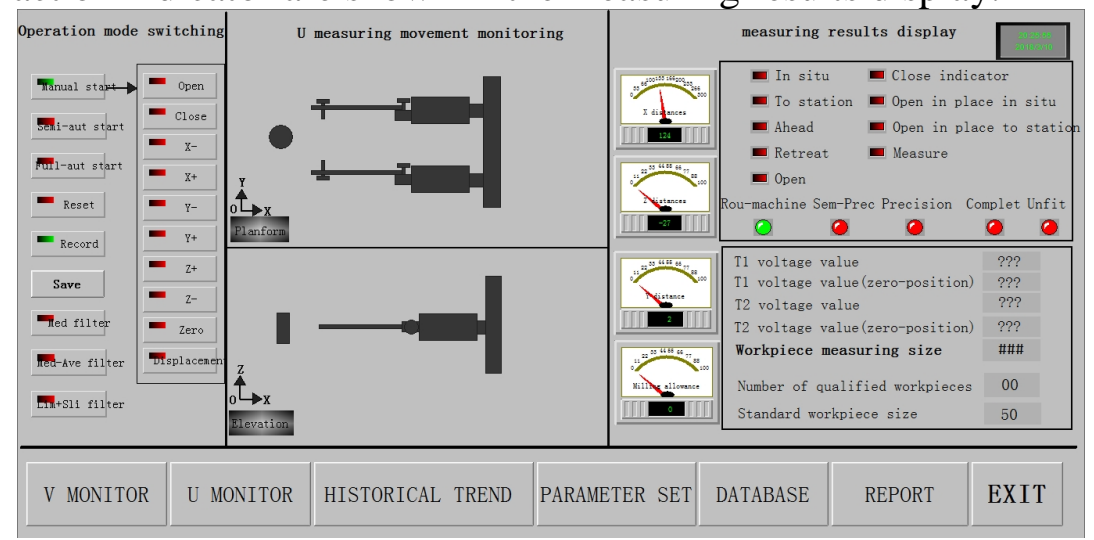

Fig.5. Interface of Unimar Probe Monitoring Module

\section{Workpiece quality statistic analysis}

The function of quality statistical analysis was realized by connecting the Access database, and the database was composed by two functional modules of data entry and data inquiry, the interface of database is shown in Fig. 6. The detection data and relevant information of the key dimensions of the batch workpiece were saved into the database by the data entry module. For example, the milling allowance of the workpiece and the number of qualified workpieces were set as the detection value, date and time are set as relevant information. The function of data single query, full query and 
conditional query were primarily done by the data query module, in addition, the data reports obtained by full query or conditional query can be printed or save. And the historical trend curve and report were designed to monitor the quality of batch workpiece.

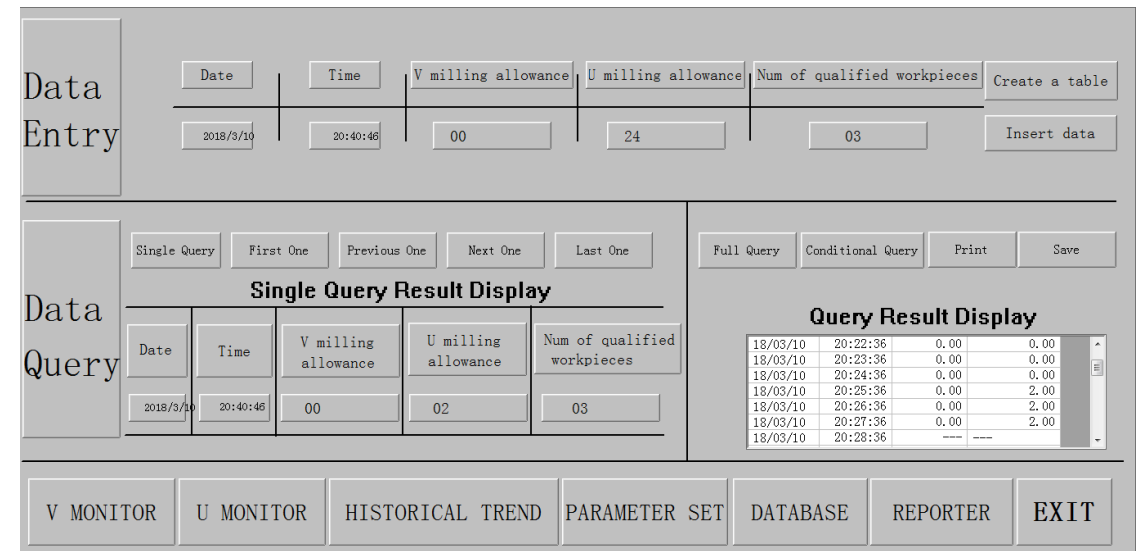

Fig.6. Interface of Database

\section{Conclusions}

In this paper, based on the study of the working principle, process flow and the monitoring technology of measuring state of the CNC pneumatic measuring instrument, the configuration collaborative method was introduced into the measuring instrument monitoring system.

CNC pneumatic measurement instruments monitoring system was constructed by KINGVIEW and PLC, which can realize the real-time monitoring and controlling of measuring probe in manual mode, semi-automatic mode and full-automatic mode, overtravel and collision warning, procedure allowance feedback controlling, control mode of switching and workpiece quality statistical analysis, and so on. The dynamics and flexibility of the monitoring system, the interactivity of all parts of the monitoring system and production were all improved. In addition, the automation and integration of machining, detection and quality control were realized.

At the same time, it laid the foundation for the improvement of the intelligent level of the measuring instrument and the realization of the digital workshop, and the following can be focused on the intelligent monitoring algorithm of the measuring instrument.

\section{Acknowledgements}

This work is supported by National Natural Science Foundation of China (Grant No. 51305124), Natural Science Foundation of Tianjin (No. 16JCYBJC19100), Natural Science Foundation of Hebei Province (No. E2017202294) and Key Research Projects of Hebei(16211803D).

\section{References}

[1] C.Yin, F.Zhang,X.B.Li,et al:Comput Integr Manuf.Sci.Forum Vol. 19 (2013),p.46.

[2] P. NOVAK, R. SINDELAR and R .MORDINYI. Simul Model Pract Th.Sci. Forum Vol. 47(201 4),p121.

[3] Z.Q.Yang and J.J.Meng.Computer Engineering and Applications.Forum Vol. 43(2007),p222.

[4] M.S.Wang,H.Y.Ma.Transactions of China Electrotechnical Society.Forum Vol. 30(2015),p484.

[5] G.R.Biswal, R.P. Maheshwari and M.L.Dewal. Int J Elec Power.Forum Vol. 43(2012),p162.

[6] X.E. Chen, L.Wang. Procedia Earth and Planetary Science.Forum Vol.2 (2011),p171.

[7] I.Morsi and L.M.El-Din.Measurement, Forum Vol. 47(2014),p5.

[8] Z.Q.Yang, M.Fan,L.B and Liu,et al.China. Patent 201621484669.3. (2017)

[9] P.Zheng,L.N.Zhang and W.F.Liu,et al.Chinese Journal of Scientific Instrument.Forum Vol.34 ( 2013),p2365. 
[10] W.M. He,S.Q.Song and Y.Gan,et al.Chinese Journal of Scientific Instrument, Forum Vol.35( 2014),p504. 